\title{
Radiotherapy cannot prolong overall survival of young prostate cancer patients with bone metastases
}

Bo Peng ${ }^{3,4}$, Cheng Yang $2,4^{*}$ and Jian $\mathrm{He}^{1 *}$

\begin{abstract}
Background: Patients with prostate cancer is commonly diagnosed with bone metastases. With the growing use of prostate-specific antigen testing, the frequency of prostate cancer has progressively increased in patients younger than 70 years. Radiotherapy is recognized for its effect on local control of bone metastases, but whether it could prolong overall survival is still controversial.

Methods: A total of 113 prostate cancer patients ( $<70 \mathrm{y})$ with bone metastases were retrospectively analyzed. The Kaplan-Meier method was used for survival analysis with log-rank test. Multivariate analysis was performed to find the prognostic factors with the COX regression model.

Results: The 1-, 2-, 3-, 5-, 7- and 10-year survival rates were $97.14,82.86,62.61,38.76,25.83$ and $13.84 \%$ respectively in the radiotherapy group, and $92.75,73.91,54.66,36.63,26.03$ and $17.85 \%$ respectively in the non-radiotherapy group, which showed no significant difference. Multivariate COX regression showed the overall survival was associated with alkaline phosphatase when bone metastases occurred and the number of bone metastases.
\end{abstract}

Conclusion: With the advances in life-prolonging treatment of metastatic prostate cancer, radiotherapy may not be the first choice for young bone metastatic prostate cancer patients in order to improve survival.

Keywords: Radiotherapy, Prostate cancer, Bone metastaese

\section{Background}

Prostate cancer $(\mathrm{PCa})$ is the most commonly diagnosed malignant tumor among males in developed countries and the second leading cause of cancer-related mortality [1]. Although PCa is usually considered as a disease of advanced age, its frequency has progressively increased in patients younger than 70 years with the growing use of prostate-specific antigen (PSA) testing [2]. Autopsy series on patients with PCa reveal that $80-85 \%$ of them have bone metastasis, with the pelvis and the vertebrae being involved in nearly all cases [3]. Although $\mathrm{PCa}$ prefers to metastasize to skeleton, patients with $\mathrm{PCa}$ in

\footnotetext{
*Correspondence: esuperyc@163.com; hejian62@163.com

1 Department of Radiation Oncology, Zhongshan Hospital, Fudan University, 180 Fenglin Road, Shanghai 200032, China

2 Department of Plastic Surgery, Zhongshan Hospital, Fudan University, 180 Fenglin Road, Shanghai 200032, China

Full list of author information is available at the end of the article
}

whom bone metastasis develops have a relatively good survival prognosis, which may even be a few years. Therefore, local control of bone metastasis for patients with prostate cancer is more important than patients with other cancers.

Radiotherapy (RT) is considered as an effective treatment for local control of bone metastasis, which could reduce the skeletal-related events (SREs) $[4,5]$. It is estimated that the mean cost for RT on bone metastases is as high as 7553 USD per episode in the United States, which has become a heavy burden for national health care system [6]. However, the long-term effect of RT on bone metastases is still controversial. In this retrospective study, we reviewed 113 young PCa patients $(<70 \mathrm{y})$ who had bone metastasis. Patients' characteristics, treatment efficacy, and prognosis were analyzed. The result revealed that RT on bone metastases cannot prolong overall survival of young PCa patients. 


\section{Patients and methods}

\section{Patient collection}

A total of 113 patients $(<70 y)$ with bone metastases from PCa who were treated between 1997 and 2012 at Zhongshan Hospital, Fudan University, were included. All of the patients were confirmed with primary $\mathrm{PCa}$ by pathological diagnosis and were diagnosed with bone metastasis through radioisotope scanning or magnetic resonance imaging.

\section{Collection of clinic pathological data}

Patient age at diagnosis, Gleason score at initial diagnosis, treatment for primary prostate lesions, number of bone lesions, PSA and alkaline phosphatase (ALP) levels, organ metastases sites, regional and remote lymph node metastases, as well as follow-up duration and survival status, were retrospectively collected and reviewed. Serum PSA and ALP values were determined in the department of clinical laboratory of Zhongshan Hospital. The PSA values of the patients were characterized as 0-4 $\mathrm{ng} / \mathrm{ml}, 4-20 \mathrm{ng} / \mathrm{ml}$ or more than $20 \mathrm{ng} / \mathrm{ml}$, while the ALP values were characterized as less than $150 \mathrm{U} / \mathrm{l}$, equal or more than $150 \mathrm{U} / \mathrm{l}$. In the pathological examinations of the patients, Gleason scores were characterized as 2-4, $5-7$ or $8-10$. When determining the number of metastases by radioisotope scanning, the number of metastases in each vertebra and rib was calculated as one; in the statistical evaluation, the number of bone metastases was assessed as either single or multiple.

\section{Radiotherapy for bone metastases}

Indications of RT for bone metastases included pain, risk of pathologic fracture, and neurologic complications arising from spinal cord compression and nerve root pain. For patients with multiple bone metastases, those with lesions causing pain or possibly spinal cord compression were first considered for RT. Bone metastatic status was recorded at the initial $\mathrm{RT}$ session for metastatic bone disease. Irradiation was delivered through a single posterior field or parallel opposed fields, depending on the location and depth of lesions according to CT or MRI. The majority of therapy was provided with 6-megavolt (MV) or 15-MV photons; however, electron therapy was also selected for those with shallow lesions such as in the ribs or skull, or extremity metastases. Radiation fields involved macroscopic tumor volume and 1 to $1.5 \mathrm{~cm}$ margins. In the case of vertebral bone metastases, radiation fields usually encompassed 1 normal vertebra above and below the metastatic lesions. If the lesions presented with soft-tissue extension concurrence, the radiation fields were enlarged on the basis of CT or MRI results. We scheduled the full radiation dosage at 46 Gy for the vertebral metastatic lesions and 50-60 Gy for soft-tissue concurrence beyond the spinal cord, in daily doses of $2 \mathrm{~Gy} /$ fraction, 5 times a week. However, factors that indicated the need for a reduced dose were considered, such as progressive primary disease, many lesions, poor Karnofsky performance status, adverse effects, and patient inconvenience during RT.

\section{Follow-up and statistical methods}

There were 5 in the non-RT group and 4 patients in the RT group lost to follow-up. The survival time was defined as from the date the first diagnosis of bone metastases to the date of death or of the last follow-up. The univariate and multivariate analyses were performed using SPSS 18.0 software (IBM, Armonk, NY, USA). The KaplanMeier method with a log-rank test was used for survival rate calculations and to evaluate each variable. Multivariate analysis was carried out with the Cox regression model, and all of the variables were analyzed with the method "enter". All of the tests were two-sided, and $p<0.05$ was considered statistically significant.

\section{Results}

\section{Patient characteristics}

A total of 104 patients $(92.0 \%)$ were followed up until the date of death or 30 June 2015. The average follow-up time for all patients was 50.01 months $(\mathrm{SD}=32.94)$ while the median was 41 months (range from 1 to 177 months). All of the patients received endocrine therapy, including surgical castration, androgen ablation, or maximal androgen blockade. Among them, 35 patients received radiotherapy on bone metastases and 69 patients did not. The age, Gleason score, PSA level, ALP level, rate of receiving chemotherapy, the number of bone metastases, or other organ metastasis showed no significant difference between the RT and non-RT group. Only the rate of regional node metastasis showed significant difference. The baseline characteristic data are shown in Table 1.

\section{Bone metastasis sites}

Vertebrae, ribs and pelvises were the most common metastasis sites in PCa patients. Figure 1 shows bone metastatic sites in patients.

\section{Survival rate analysis}

From the diagnosis of bone metastases, the 1-, 2-, 3-, 5-, 7 - and 10-year survival rates were 97.14, 82.86, 62.61, $38.76,25.83$ and $13.84 \%$ respectively in the RT group, and $92.75,73.91,54.66,36.63,26.03$ and $17.85 \%$ respectively in the non-RT group. The survival rate of RT and non-RT group showed no significant difference (Table 2 and Fig. 2).

As the significant difference of regional lymph node metastasis between RT and non-RT group, stratified 
Table 1 Baseline characteristics of patients

\begin{tabular}{|c|c|c|c|}
\hline & RT & Non-RT & $P$ value \\
\hline Age & $64.26 \pm 4.98$ & $63.96 \pm 5.48$ & 0.788 \\
\hline Gleason score & $7.91 \pm 1.31$ & $7.88 \pm 1.33$ & 0.913 \\
\hline PSA & $323.05 \pm 529.83$ & $368.14 \pm 613.40$ & 0.713 \\
\hline ALP & $304.32 \pm 455.35$ & $316.52 \pm 346.32$ & 0.884 \\
\hline Chemotherapy & & & 0.162 \\
\hline Yes & $9(25.71 \%)$ & $10(14.49 \%)$ & \\
\hline No & $26(74.29 \%)$ & $59(85.51 \%)$ & \\
\hline The number of bone metastases & & & 0.975 \\
\hline Single & $6(17.14 \%)$ & $12(17.39 \%)$ & \\
\hline Multiple & $29(82.86 \%)$ & $57(82.61 \%)$ & \\
\hline Regional lymph node metastasis & & & 0.002 \\
\hline Yes & $1(2.86 \%)$ & $20(28.99 \%)$ & \\
\hline No & $34(97.14 \%)$ & $49(71.01 \%)$ & \\
\hline Other organ metastasis & & & 0.09 \\
\hline Yes & $7(20.00 \%)$ & 25 (36.23 \%) & \\
\hline No & 28 (80.00 \%) & 44 (63.77 \%) & \\
\hline
\end{tabular}

$P S A$ prostate-specific antigen; $A L P$ alkaline phosphatase; $R T$ radiotherapy

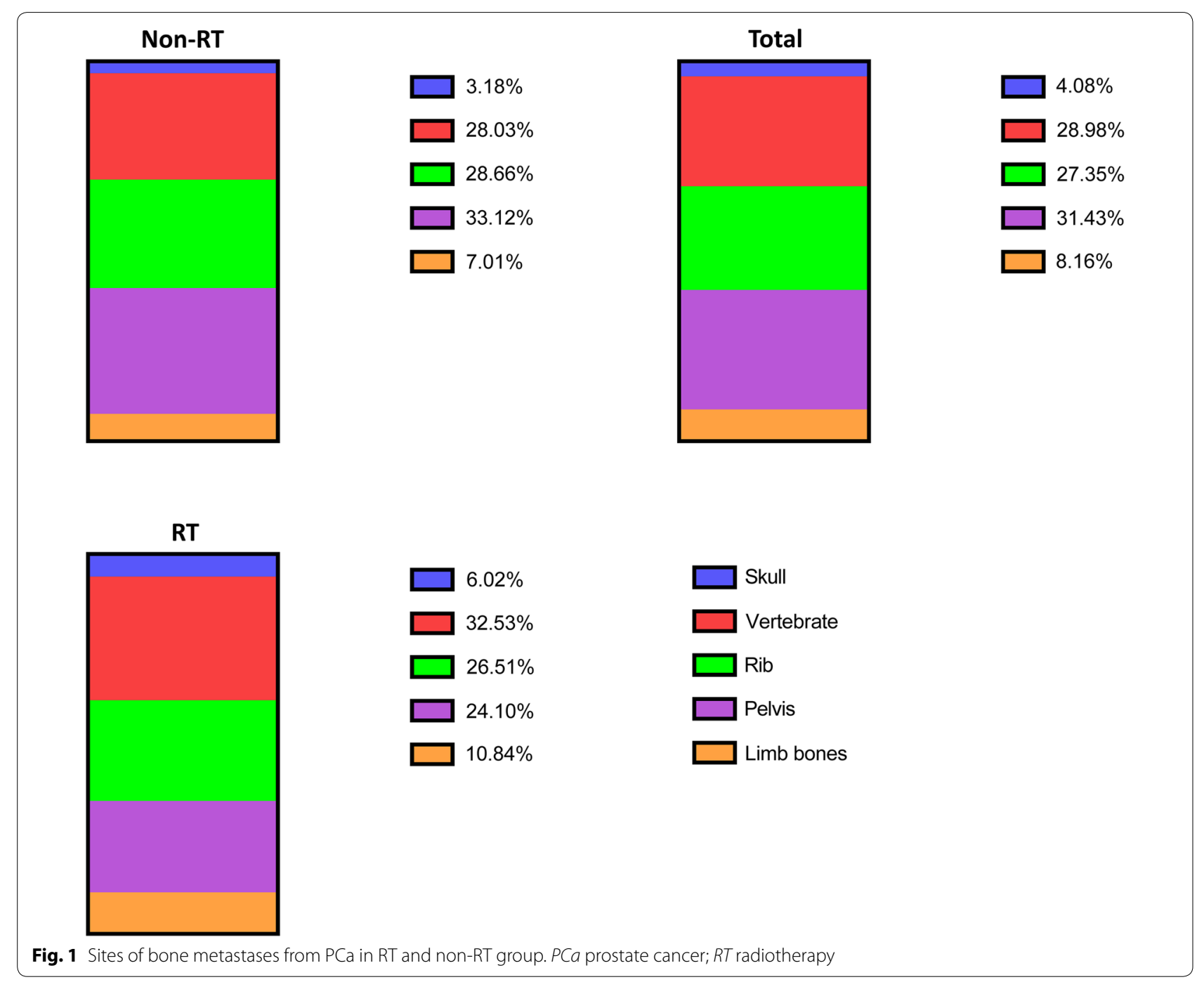


Table 2 Univariate analysis for survival

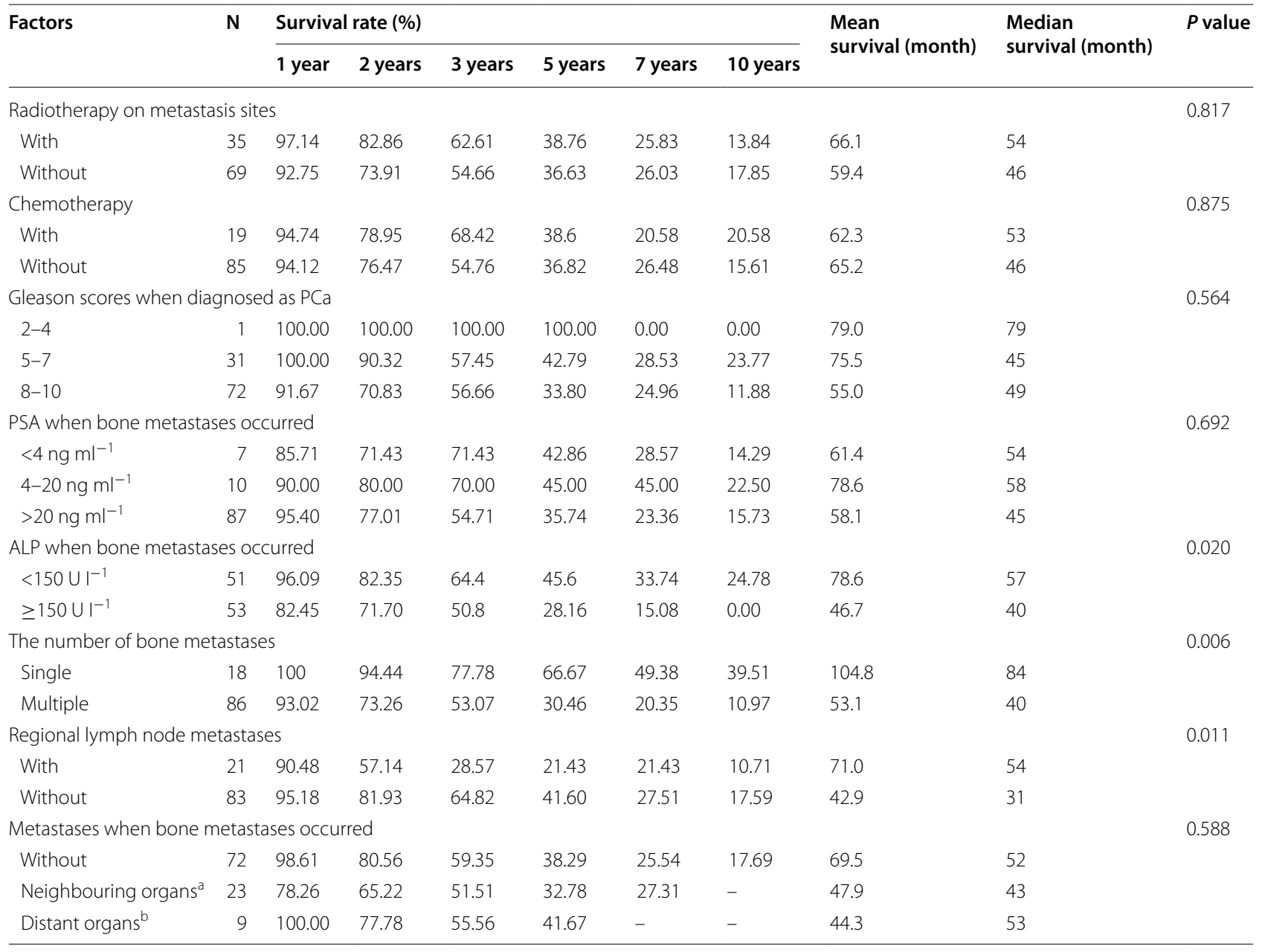

ALP alkaline phosphatase; PSA prostate-specific antigen

a Defined as an organ around with prostate, such as the bladder, spermatophores, urethra and rectum

b Defined as brain, lung and liver metastases

analysis was performed. When adjusted for this factor, there was still no significant difference between the RT and non-RT groups (Table 3 ).

\section{Prognostic factor analysis}

The univariate analysis of the results indicated that the survival was associated with ALP when bone metastases occurred, the number of bone metastases, and regional lymph node metastases when bone metastases occurred $(p<0.05)$. The differences for other factors were not statistically significant ( $p>0.05$, Table 2 and Fig. 2).

Multivariate Cox regression analysis indicated that ALP when bone metastases occurred and the number of bone metastases were significant factors of survival, while the radiotherapy was not. The coefficient of regression and relative risk were showed in Table 4.

\section{Discussion}

The clinical features of bone metastases from PCa are similar with other malignancies in which bone metastases most commonly affect the axial skeleton [7]. The most common metastatic sites are the vertebrae, ribs and pelvis. RT is one of the most important therapeutic options available in advanced PCa stage. Its effects have been recognized for local control of bone metastases and reducing the SREs. However, when it comes to the longterm prognosis of RT for PCa bone metastases, it is still controversial [8-11].

Conventionally, RT is just a palliative treatment for advanced PCa patients with much lower doses to destroy the tumor cells. The exact mechanism of palliative RT has not been well defined, which may be the effects on bone homeostasis or alteration of signaling pathways $[8,12]$. 

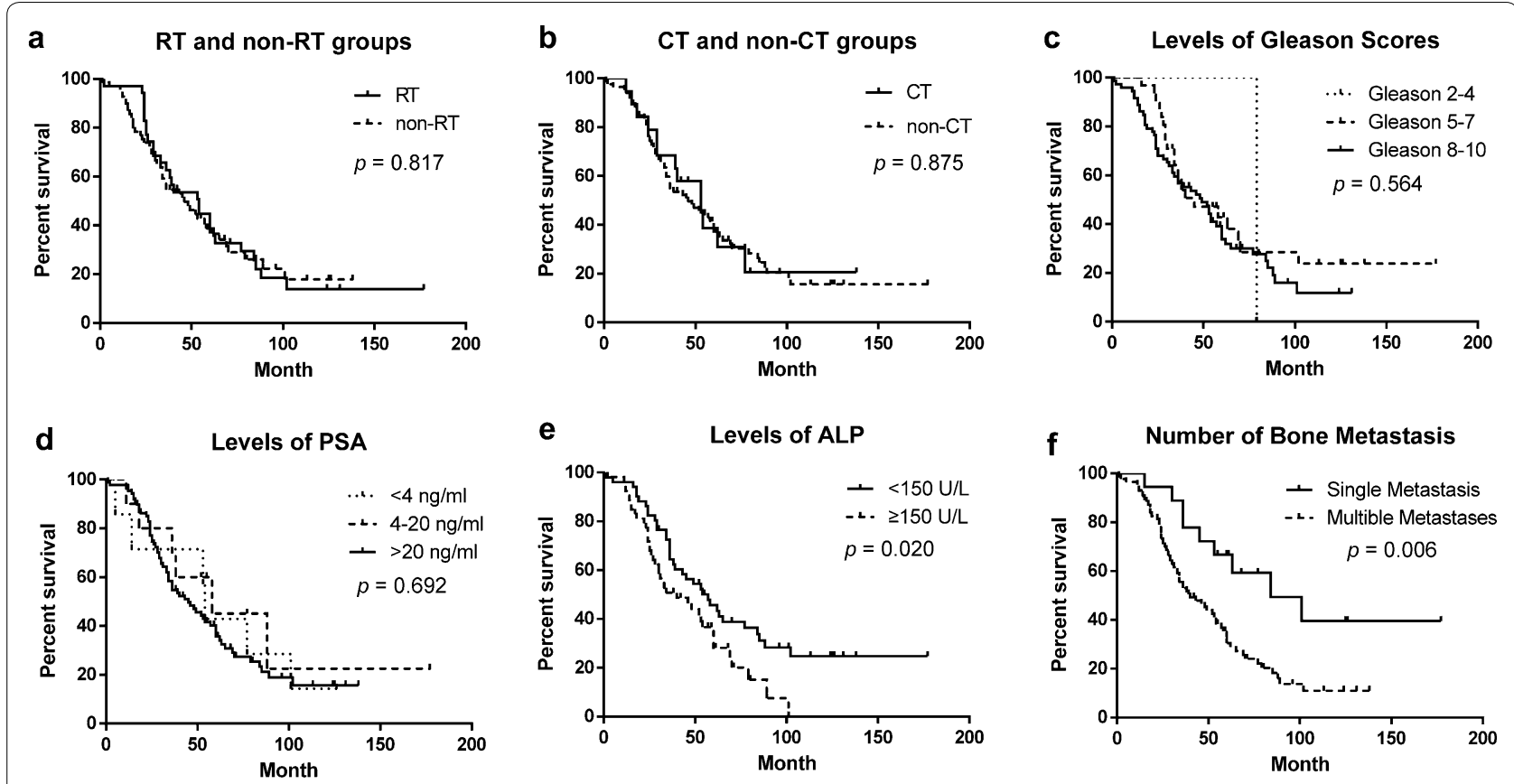

g Metastases of Reginal Lymph Node
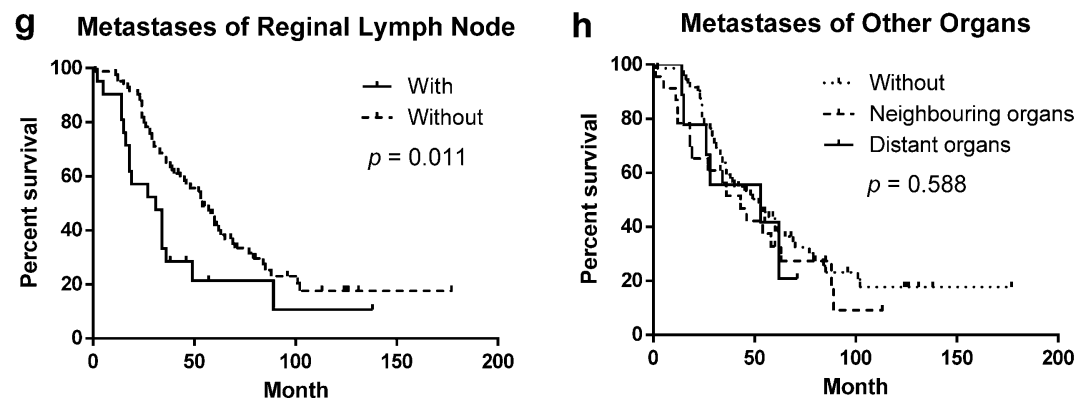

Fig. 2 The overall survival curves of patients with Kaplan-Meier estimator, tested with a log-rank test. a The overall survival curves of RT and non-RT groups, $p=0.817$. b The overall survival curves of CT and non-CT groups, $p=0.875$. c The overall survival curves of patients with different levels of Gleason scores, $p=0.564$. d The overall survival curves of patients with different levels of PSA, $p=0.692$. e The overall survival curves of patients with different levels of ALP, $p=0.020$. $\mathbf{f}$ The overall survival curves of patients with single or multiple bone metastases, $p=0.006$. $\mathbf{g}$ The overall survival curves of patients with or without metastases of reginal lymph nodes, $p=0.011$. $\mathbf{h}$ The overall survival curves of patients with or without metastases of other organs, $p=0.588$. $R T$ radiotherapy; $C T$ chemotherapy; $P S A$ prostate-specific antigen; $A L P$ alkaline phosphatase

Table 3 Stratified analysis of radiotherapy

\begin{tabular}{lccll}
\hline $\begin{array}{l}\text { Regional lymph } \\
\text { node } \\
\text { metastases }\end{array}$ & $\mathbf{N}$ & $\begin{array}{l}\text { Mean } \\
\text { survival } \\
\text { (month) }\end{array}$ & $\begin{array}{l}\text { Median } \\
\text { survival } \\
\text { (month) }\end{array}$ & $\begin{array}{l}\text { Adjusted } \\
\boldsymbol{p} \text { value }\end{array}$ \\
\hline With & & & & \\
RT & 1 & 2.0 & 2 & 0.596 \\
Non-RT & 20 & 45.0 & 31 & \\
Without & & & & \\
RT & 34 & 68.0 & 54 & \\
Non-RT & 49 & 63.5 & 57 & \\
\hline
\end{tabular}

Comparing with the radical prostatectomy, which dose is recommended for greater than $70 \mathrm{~Gy}$, the procedure of palliative RT is commonly a single-fraction dose (4-8 Gy) or multi-fraction higher doses (20-45 Gy) [8-10, 13]. In our study, the full radiation dose for the patients is $46 \mathrm{~Gy}$, and part of the patients had less dose due to side effects and other reasons. It is demonstrated that the dosage and schedule of RT is associated with the overall survival of PCa patients with bone metastasis. Tabata et al. [11] had shown that the oligometastases and oligo recurrence of bone metastases in PCa patients treated with 
Table 4 Multivariate analysis for survival

\begin{tabular}{|c|c|c|c|c|}
\hline Factors & $\beta$ & S.e. & RR & $P$ value \\
\hline Radiotherapy on metastasis sites & 0.209 & 0.289 & 1.233 & 0.469 \\
\hline Chemotherapy & -0.198 & 0.316 & 0.820 & 0.530 \\
\hline Gleason scores when diagnosed as Pca ${ }^{a}$ & & & & 0.876 \\
\hline $2-4$ & -0.507 & 1.037 & 0.602 & 0.625 \\
\hline $5-7$ & -0.054 & 0.277 & 0.948 & 0.846 \\
\hline PSA when bone metastases occurred ${ }^{b}$ & & & & 0.569 \\
\hline$<4 \mathrm{ng} \mathrm{ml}^{-1}$ & 0.492 & 0.475 & 1.635 & 0.301 \\
\hline $4-20 \mathrm{ng} \mathrm{m}^{-1}$ & 0.214 & 0.489 & 1.239 & 0.661 \\
\hline ALP when bone metastases occurred & 0.461 & 0.251 & 1.586 & 0.041 \\
\hline The number of bone metastases & 0.889 & 0.410 & 2.433 & 0.030 \\
\hline Regional lymph node metastases & 0.580 & 0.321 & 1.786 & 0.071 \\
\hline $\begin{array}{l}\text { Metastases when bone metastases } \\
\text { occurred }^{c}\end{array}$ & & & & 0.957 \\
\hline Without & -0.108 & 0.443 & 0.897 & 0.807 \\
\hline Neighbouring organs & -0.050 & 0.511 & 0.951 & 0.922 \\
\hline
\end{tabular}

$A L P$ alkaline phosphatase; $\beta$ coefficient of regression; $P C a$ prostate cancer; $R R$ relative risk; s.e. standard error

a Gleason 8-10 used as control group

b PSA > $20 \mathrm{ng} \mathrm{ml-1}$ used as control group

c Distant organs used as control group

conventional RT doses of $>40$ Gy had a superior 3-year survival compared with those treated with $<40$ Gy $(90.5$ vs $50.0 \%, p=0.012)$. Wu et al. had also indicated that the long-course RT (37.5 Gy in 15 fractions, 40 Gy in 20 fractions, or $50 \mathrm{~Gy}$ in 25 fractions) had a better 3-year overall survival than short-course RT (20 Gy in 5 fractions or $30 \mathrm{~Gy}$ in 10 fractions) in bone oligometastases PCa patients underwent $\mathrm{RT}$ combined with endocrine therapy after curative RT for PCa (76.4 vs $44.1 \%, p=0.03$ ) [14]. In our study, that the survival between RT and non-RT group showed no significant difference may be the result of insufficient dose of RT.

Another critical factor that influences the result is the sites for RT. As mentioned in methods, for patients with multiple bone metastases, those with lesions causing pain or possibly spinal cord compression were first considered for RT. Not all lesions received radiation so that it might influence the long-term survival. To have better effect on the diffuse lesions, radionuclides were introduced for the treatment to bone metastatic cancer. Several radionuclides have shown effects on the relief of SREs, like strontium-89, samarium-153 and radium-223. Among them, radium-223 is the most promising radionuclide that could improve the survival [15]. Radium-223 is a bone-seeking calcium mimetic, which selectively binds to areas of increased bone turnover in bone metastases, and emits high-energy alpha particles of short range $(<100 \mu \mathrm{m})$ [16]. A phase 3, randomized, double-blind, placebo-controlled study conducted by Parker et al. [17] showed that radium-223 significantly improved overall survival of bone metastatic PCa patients compared with placebo (14.0 months vs. 11.2 months; HR 0.70; $95 \%$ CI $0.55-0.88 ; p=0.002)$. The study was terminated for efficacy at the interim analysis and the result was published on the NEJM.

In our study, the number of bone metastases, the ALP when bone metastases occurred and regional lymph node metastases were the prognostic factors analyzed by univariate analysis. When adjusting for confounding variables, only the number of bone metastases and the ALP when bone metastases occurred had significant difference. The number of bone metastases had a strong impact on the survival, which was in agreement with previous studies. Singh et al. [18] reported that patients with five or less metastatic lesions had higher 5-year overall survival compared with those with five lesions (73 vs $45 \%$ ), which was similar to metastasis-free patients. Schick et al. reported a 3-year biochemical recurrencefree survival of $66.5 \%$ in patients treated with androgen deprivation combined with high-dose RT for only one metastatic lesion and $36.4 \%(p=0.031)$ in those treated for more than one metastases [19]. Similarly, another study by Wu et al. [14] showed that significantly improved three-year overall survival was observed in PCa patients with one metastatic lesion compared with patients with more than one metastatic lesions (78.8 vs $42.2 \%$ ). For patients with single or limited bone metastases, they may get better prognosis with aggressive RT. Therefore, Hellman and Weichselbaum firstly hypothesized that the local treatment of patients with limited number of metastatic or recurrent lesions, using surgical resection and RT, improved systemic control. In this situation, the number of metastases was less than five, and the primary lesion could be controlled [20]. It further arose the viewpoint for restaging the stage IV cancer. Rubin suggested amending the TNM staging system, modifying the " $\mathrm{M}$ " to represent solitary metastasis (M1), oligometastases (M2), or multiple metastases (M3) [21]. For oligometastases of $\mathrm{PCa}$, it means a controlled or controllable primary lesion with five or fewer metastases (ideally 1-3) located in the bone (preferably the spine, or ganglions) [22]. With a few retrospective studies, it was suggested that local therapy to a small number of gross metastatic sites and recurrences might result in prolonged survival or even cure $[14,19,20,22,23]$. Several phase II prospective clinic trials are seeking for more convincing evidence for the treatment to oligometastases of $\mathrm{PCa}[22,24]$.

With the advances in the treatment of metastatic $\mathrm{PCa}$, the viewpoint has changed from palliative treatment to life-prolonging treatment. Several agents have shown effects on the improvement of survival for metastatic PCa patients, including docetaxel with prednisone, 
cabazitaxel with prednisone, Sipuleucel-T, abiraterone with prednisone, enzalutamide and radium-223 [25]. In order to prolong the survival of the metastatic PCa patients, radiotherapy may not be the first choice. However, for palliative treatment only, single fraction of RT is still considered as an effective and cost-effective method $[5,26,27]$. Therefore, the choice of treatment strategy should be considered thoroughly based on the state of the patients and the objective of the treatment.

\section{Conclusion}

In conclusion, RT on the bone metastases cannot prolong the overall survival of young PCa patients. The number of bone metastases and the ALP when bone metastases occurred are the prognostic factors. In order to improve the survival of metastatic PCa patients, RT may not be the first choice.

\section{Authors' contributions}

$\mathrm{CY}$ and $\mathrm{JH}$ conceived and designed the study. BP collected, analyzed patients data, and wrote the draft. CY revised the manuscript. All authors read and approved the final manuscript.

\section{Author details \\ 1 Department of Radiation Oncology, Zhongshan Hospital, Fudan University, 180 Fenglin Road, Shanghai 200032, China. ${ }^{2}$ Department of Plastic Surgery, Zhongshan Hospital, Fudan University, 180 Fenglin Road, Shanghai 200032, China. ${ }^{3}$ Department of Urology, Zhongshan Hospital, Fudan University, 180 Fenglin Road, Shanghai 200032, China. ${ }^{4}$ Shanghai Key Laboratory of Organ Transplantation, Shanghai, China.}

\section{Acknowledgements}

This study was supported by National Natural Science Foundation of China (Grants 81400752 to $C Y$ )

\section{Competing interests}

The authors declare that they have no competing interests.

\section{Ethics, consent and permissions}

The study design was approved by the institutional ethics review board of Zhongshan Hospital, Fudan University.

Received: 10 March 2016 Accepted: 13 April 2016

Published online: 27 April 2016

\section{References}

1. Siegel RL, Miller KD, Jemal A. Cancer statistics, 2015. CA Cancer J Clin. 2015;65(1):5-29.

2. Welch $\mathrm{HG}$, Albertsen PC. Prostate cancer diagnosis and treatment after the introduction of prostate-specific antigen screening: 1986-2005. J Natl Cancer Inst. 2009;101(19):1325-9.

3. Jacobs SC. Spread of prostatic cancer to bone. Urology. 1983:21(4):337-44.

4. Ost P, Bossi A, Decaestecker K, De Meerleer G, Giannarini G, Karnes RJ, Roach M 3rd, Briganti A. Metastasis-directed therapy of regional and distant recurrences after curative treatment of prostate cancer: a systematic review of the literature. Eur Urol. 2015:67(5):852-63.

5. Rutter CE, Yu JB, Wilson LD, Park HS. Assessment of national practice for palliative radiation therapy for bone metastases suggests marked underutilization of single-fraction regimens in the United States. Int J Radiat Oncol Biol Phys. 2015:91(3):548-55.
6. Yong C, Onukwugha E, Mullins CD. Clinical and economic burden of bone metastasis and skeletal-related events in prostate cancer. Curr Opin Oncol. 2014;26(3):274-83.

7. He J, Zeng ZC, Tang ZY, Fan J, Zhou J, Zeng MS, Wang JH, Sun J, Chen $B$, Yang $P$, et al. Clinical features and prognostic factors in patients with bone metastases from hepatocellular carcinoma receiving external beam radiotherapy. Cancer. 2009:115(12):2710-20.

8. Rove KO, Crawford ED. Evolution of treatment options for patients with CRPC and bone metastases: bone-targeted agents that go beyond palliation of symptoms to improve overall survival. Oncology (Williston Park). 2011:25(14):1362-1370, 1375-1381, 1387.

9. Pinkawa M. External beam radiotherapy for prostate cancer. Panminerva Med. 2010;52(3):195-207.

10. Boyer MJ, Salama JK, Lee WR. Palliative radiotherapy for prostate cancer. Oncology (Williston Park). 2014;28(4):306-12.

11. Tabata K, Niibe Y, Satoh T, Tsumura H, Ikeda M, Minamida S, Fujita T, Ishii D, Iwamura M, Hayakawa K, et al. Radiotherapy for oligometastases and oligo-recurrence of bone in prostate cancer. Pulm Med. 2012;2012:541656.

12. Valkenburg KC, Steensma MR, Williams BO, Zhong Z. Skeletal metastasis: treatments, mouse models, and the Wnt signaling. Chin J Cancer. 2013:32(7):380-96.

13. Bauman G, Rumble RB, Chen J, Loblaw A, Warde P. Members of the IIEP: intensity-modulated radiotherapy in the treatment of prostate cancer. Clin Oncol (R Coll Radiol). 2012;24(7):461-73.

14. Wu JX, Lin LM, He JY, Hong L, Li JL. Radiotherapy combined with androgen deprivation for bone oligometastases after primary curative radiotherapy for prostate cancer: a retrospective study. Medicine (Baltimore). 2016:95(6):e2789.

15. Autio KA, Morris MJ. Targeting bone physiology for the treatment of metastatic prostate cancer. Clin Adv Hematol Oncol. 2013;1 1(3):134-43.

16. Bruland OS, Nilsson S, Fisher DR, Larsen RH. High-linear energy transfer irradiation targeted to skeletal metastases by the al pha-emitter 223Ra: adjuvant or alternative to conventional modalities? Clin Cancer Res. 2006;12(20 Pt 2):6250s-7s.

17. Parker C, Nilsson S, Heinrich D, Helle SI, O'Sullivan JM, Fossa SD, Chodacki A, Wiechno $P$, Logue J, Seke M, et al. Alpha emitter radium-223 and survival in metastatic prostate cancer. N Engl J Med. 2013;369(3):213-23.

18. Singh D, Yi WS, Brasacchio RA, Muhs AG, Smudzin T, Williams JP, Mess-

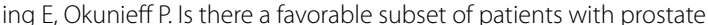
cancer who develop oligometastases? Int J Radiat Oncol Biol Phys. 2004:58(1):3-10.

19. Schick U, Jorcano S, Nouet P, Rouzaud M, Vees H, Zilli T, Ratib O, Weber DC, Miralbell R. Androgen deprivation and high-dose radiotherapy for oligometastatic prostate cancer patients with less than five regional and/ or distant metastases. Acta Oncol. 2013:52(8):1622-8.

20. Hellman S, Weichselbaum RR. Oligometastases. J Clin Oncol. 1995;13(1):8-10.

21. Rubin P, Brasacchio R, Katz A. Solitary metastases: illusion versus reality. Semin Radiat Oncol. 2006;16(2):120-30.

22. Conde Moreno AJ. Ferrer Albiach C, Muelas Soria R, Gonzalez Vidal V, Garcia Gomez R, Albert Antequera M: oligometastases in prostate cancer: restaging stage IV cancers and new radiotherapy options. Radiat Oncol. 2014; $9: 258$

23. Niibe Y, Hayakawa K. Oligometastases and oligo-recurrence: the new era of cancer therapy. Jpn J Clin Oncol. 2010;40(2):107-11.

24. Decaestecker K, De Meerleer G, Ameye F, Fonteyne V, Lambert B, Joniau S, Delrue L, Billiet I, Duthoy W, Junius S, et al. Surveillance or metastasisdirected therapy for oligometastatic prostate cancer recurrence (STOMP): study protocol for a randomized phase II trial. BMC Cancer. 2014;14:671.

25. Park JC, Eisenberger MA. Advances in the treatment of metastatic prostate cancer. Mayo Clin Proc. 2015:90(12):1719-33.

26. Hess G, Barlev A, Chung K, Hill JW, Fonseca E. Cost of palliative radiation to the bone for patients with bone metastases secondary to breast or prostate cancer. Radiat Oncol. 2012;7:168.

27. Konski A. Radiotherapy is a cost-effective palliative treatment for patients with bone metastasis from prostate cancer. Int J Radiat Oncol Biol Phys. 2004:60(5):1373-8. 